\title{
The Rotational Zeeman Effect in Fluorobenzene and the Molecular Quadrupole Moment in Benzene
}

\author{
W. H. Stolze, M. Stolze, D. Hübner, and D. H. Sutter \\ Institut für Physikalische Chemie, Abt. Chemische Physik, Universität Kiel
}

Z. Naturforsch. 37a, 1165-1175 (1982); received June 14, 1982

In memory of W.H. Flygare who opened the field

\begin{abstract}
The rotational Zeeman effect in fluorobenzene is reinvestigated with a resolution improved by a factor of almost five to give more accurate $g$-tensor elements, magnetic susceptibility anisotropies and molecular electric quadrupole moments. The results fit into the pictures of a linear dependence of the out of plane molecular electric quadrupole moment, $Q_{c c}$, on the number of fluorine substituents and of a linear correlation between the nonlocal (ring current) susceptibility, $\chi_{c c}^{\text {nonlocal }}$, and the CNDO/2- $\pi$-electron density alternation. They lead to a gasphase molecular electric quadrupole moment in benzene, $Q_{c c}$, benzene $=-(28.4 \pm 4.7) \cdot 10^{-40} \mathrm{Cm}^{2}$ which is slightly less negative than the value deduced from electric field-gradient birefringence experiments on dilute benzene solutions with carbon tetrachloride as solvent. A detailed description of the high resolution microwave spectrometer is given in the appendix.
\end{abstract}

\section{Introduction}

The present investigation has been initiated to resolve the discrepancy between the published experimental values for the molecular quadrupole moment of benzene (for its definition in the rigid rotor model see (1)).

$$
\begin{aligned}
Q_{c c}= & \frac{e}{2} \sum_{\nu}^{\text {nuclei }} Z_{v}\left(2 c_{\nu}^{2}-b_{\nu}^{2}-a_{\nu}^{2}\right) \\
& -\frac{e}{2}\left\langle 0\left|\sum_{\varepsilon} 2 c_{\varepsilon}^{2}-b_{\varepsilon}^{2}-a_{\varepsilon}^{2}\right| 0\right\rangle,
\end{aligned}
$$

$e=$ proton charge,

$Z_{\nu}=$ atomic number of $\nu$-th nucleus,

$a_{\nu} \quad b_{\nu} \quad c_{\nu}=$ coordinates of the $v$-th nucleus and the $\begin{array}{lllll}a_{\varepsilon} & b_{\varepsilon} & c_{\varepsilon} & \varepsilon \text {-th electron referred to the molecular }\end{array}$ principal inertia axes system ( $c$-axis perpendicular to the molecular plane).

$\langle 0|| 0\rangle=$ electronic ground state expectation value.

The first quasi experimental value for the benzene quadrupole moment has been calculated by Shoemaker and Flygare [1] from the observed quadrupole moments in fluorobenzene [2] according to

$$
\begin{aligned}
& Q_{c c, \text { benzene }} \\
& =\frac{2}{3}\left(Q_{c c, \text { fluorobenzene }}-Q_{b b, \text { fluorobenzene }}\right) .
\end{aligned}
$$

Reprint requests to Prof. Dr. D. H. Sutter, Institut für Physikalische Chemie, Abt. Chemische Physik, ChristianAlbrechts-Universität Kiel, Olshausenstr. 40-60, D-2300 Kiel.
(In fluorobenzene the $a$-axis coincides with the $\mathrm{C}-\mathrm{F}$ bond, $c$-axis perpendicular to the ring.)

Equation (2) is appropriate provided that two assumptions hold:

a) The substitution of the fluorine atom does not appreciably distort the nuclear framework of the benzene ring.

$$
\begin{aligned}
& \text { b) }\left\langle 0\left|\sum_{\varepsilon}^{\text {electrons }} b_{\varepsilon}^{2}-c_{\varepsilon}^{2}\right| 0\right\rangle_{\text {fluorobenzene }} \\
& =\left\langle 0\left|\sum_{\varepsilon}^{\text {electrons }} b_{\varepsilon}^{2}-c_{\varepsilon}^{2}\right| 0\right\rangle_{\text {benzene }} .
\end{aligned}
$$

Flygare and Shoemaker have discussed the validity of both assumptions and they have concluded that the uncertainty in their quadrupole moment of benzene is largely determined by the uncertainties with which the fluorobenzene $g$-values and susceptibility anisotropies were determined. Their value:

$$
\begin{aligned}
Q_{c c, \text { benzene }} & =-(5.6 \pm 2.8) \cdot 10^{-26} \mathrm{esu} \mathrm{cm}^{2} \\
& =-(18.7 \pm 9.3) \cdot 10^{-40} \mathrm{C} \mathrm{m}^{2}
\end{aligned}
$$

The second experimental value for the molecular electric quadrupole moment of benzene was determined by Vrbancich and Ritchie [3] who used the electric field-gradient birefringence method applied to dilute benzene solutions with carbon tetrachloride as solvent. Their value:

$$
\begin{aligned}
Q_{c c, \text { benzene }} & =-(9.98 \pm 0.63) \cdot 10^{-26} \mathrm{esu} \mathrm{cm}^{2} \\
& =-(33.2 \pm 2.1) \cdot 10^{-40} \mathrm{C} \mathrm{m}^{2} .
\end{aligned}
$$

0340-4811 / 82 / 1000-1165 $\$ 01.30 / 0$. - Please order a reprint rather than making your own copy. 
Since a reliable experimental value for the molecular quadrupole moment of benzene is of interest in various fields (e.g. orientational correlation in the liquid state, energy transfer in grazing collisions in the gasphase, dimer formation in nozzle beams etc.), we decided to reinvestigate the rotational Zeeman effect in fluorobenzene with considerably improved spectral resolution.

\section{Measurements and Analysis of the Data}

The high field electromagnet used in this work has been described before [4]. To improve the sensitivity and resolution, we have replaced the standard Stark. effect modulated microwave spectrograph by the Ku-band superheterodyne bridge system described in the appendix. Typical linewidth obtained were about $50 \mathrm{kHz}$ (full width at half height) as compared to about $200 \mathrm{kHz}$ in the work of Hüttner and Flygare (compare Figs. 1 and 2 of Ref. [2]) and Fig. 1 of this work). Our experimental accuracy with which the line maxima are determined is thus on the order of $\pm 2 \mathrm{kHz}$ to $\pm 5 \mathrm{kHz}$ depending on the signal to noise ratio and the experimental accuracy of the line shifts reported in Table 2 is $\pm 4 \mathrm{kHz}$ to $\pm 10 \mathrm{kHz}$.

For the analysis of the Zeeman spectra, the standard rigid rotor Hamiltonian given in (3) was used $[5,6,7]$ :

$$
\begin{aligned}
\hat{\mathscr{H}}_{\text {eff }}= & \overbrace{h\left(A \hat{J}_{a}^{2}+B \hat{J}_{b}^{2}+C \hat{J}_{c}^{2}\right)}^{\hat{\mathscr{H}}_{\text {rot }}} \\
& \overbrace{\hat{\mathscr{H}}_{\mathrm{g}} H_{Z} \sum_{\gamma} g_{\gamma \gamma} \hat{\cos \gamma Z \hat{J}_{\gamma}}}^{\underbrace{-\frac{1}{2} H_{Z^{2}} \sum_{\gamma} \chi_{\gamma \gamma} \hat{\cos } \gamma Z \hat{\cos \gamma} Z}_{\hat{\mathscr{H}}_{\chi}}},
\end{aligned}
$$

$h=$ Planck's constant,

$A, B, C=$ rotational constants

$$
\begin{aligned}
& \left(A=h / 8 \pi^{2} I_{a a}\right. \text { with } \\
& I_{a a}=\sum_{n}^{\text {atoms }} m_{n}\left(b_{n}^{2}+c_{n}{ }^{2}\right) \text { the } a \text {-th }
\end{aligned}
$$

principal moment of inertia).

$\jmath_{a}, \jmath_{b}, \jmath_{c}=$ rotational angular momentum operators along the $a$-th, $b$-th and $c$-th principal inertia axis, each divided by $\hbar$.

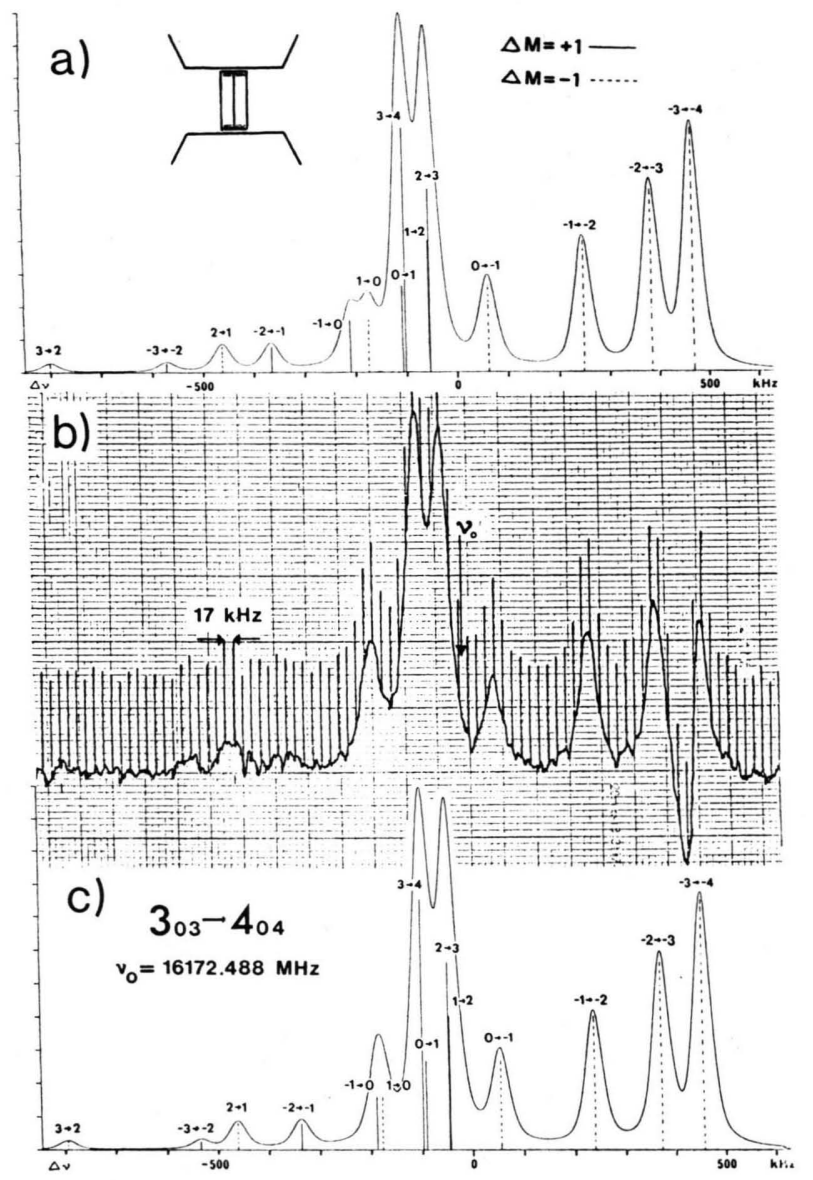

Fig. 1. Zeeman effect pattern of the $3_{03} \rightarrow 4_{04}$ rotational transition in fluorobenzene. With the electric field vector of the incident microwave radiation perpendicular to the magnetic field (see insert), the $\Delta M= \pm 1$ selection rule applies. The field is 1.8874 Tesla $=\mathbf{1 8 8 7 4}$ Gauss. Trace a) shows a computer simulation calculated from the $g$-values and susceptibility anisotropies reported by Hüttner and Flygare [2]. Trace b) shows the actual spectrum. Trace c) shows a simulation calculated with our improved values listed in Table 3. For the simulations Lorentzian lineshapes with $40 \mathrm{kHz}$ full width at half height were used. The position of the unsplit zero field line is shown by the arrow. The improvement of the data is most obvious in the $\Delta v$ region between -270 and $-140 \mathrm{kHz}$. The dent between the $-2 \rightarrow-3$ and $-3 \rightarrow-4$ satellites is due to a Stark satellite. (The absorption spectrum in the presence of the Stark field, here $1.7 \mathrm{kV} / \mathrm{cm}$, is written upside down by the phase sensitive detection scheme.) The markers are every $17 \mathrm{kHz}$.

$H_{Z}=$

magnetic field, assumed to be directed along the space fixed $Z$-axis.

$\mu_{n}=e \hbar / 2 m_{\mathrm{p}} c^{2}=$ nuclear magneton $=(5.05050 \pm$ $0.00013) \cdot 10^{-24} \mathrm{erg} /$ Gauss [8]; 
$g_{a a}, g_{b b}, g_{c c}=$ diagonal elements of the molecular $g$-tensor;

$\chi_{a a}, \chi_{b b}, \chi_{c c}=$ diagonal elements of the molecularmagnetic susceptibility tensor.

Due to the $\mathrm{C}_{2 \mathrm{v}}$-symmetry of the molecule, the moment of inertia tensor, the $g$-tensor and the $\chi$-tensor are simultaneously diagonal. Spin-rotation coupling effects and the translational Stark effect [9] can be neglected. Even with the increased resolution of our spectrometer they do not lead to measurable effects.

In (3) $\hat{\mathscr{H}}_{\text {g }}$ and $\hat{\mathscr{H}}_{\chi}$ present only a minor perturbation to the rigid rotor Hamiltonian, and the first order energy expression for the Zeeman shifts of the rigid rotor levels [10] can be used to analyse the observed Zeeman multiplets. (Second order corrections are below $0.5 \mathrm{kHz}$ for the rotational transitions studied here.) This first order energy expression is given by

$$
\begin{aligned}
& E_{J, K-K_{+}, M}=-\mu_{n} H_{Z} \frac{M}{J(J+1)} \sum_{\gamma} g_{\gamma \gamma}\left\langle\hat{J}_{\gamma^{2}}\right\rangle \\
& -\frac{1}{2} \chi H_{z}{ }^{2}-H_{z}{ }^{2} \frac{3 M^{2}-J(J+1)}{J(J+1)(2 J-1)(2 J+3)} \\
& \cdot \sum_{\gamma}\left(\chi-\chi_{\gamma \gamma}\right)\left\langle\hat{J}_{\gamma^{2}}\right\rangle .
\end{aligned}
$$

In (4) the sums run over the three principal axes $(\gamma=a, b, c), \chi=\left(\chi_{a a}+\chi_{b b}+\chi_{c c}\right) / 3$ is the average magnetic susceptibility and

$$
\left\langle\hat{J}_{\gamma^{2}}\right\rangle=\left\langle J, K_{-} K_{+}\left|\hat{J}_{\gamma^{2}}\right| J, K_{-} K_{+}\right\rangle
$$

is the rigid rotor expectation value for the squared rotational angular momentum along the $\gamma$-th principal axis divided by $\hbar^{2}$. As usual $J$ is the overall angular momentum quantum number and $M$ is the magnetic quantum number i. e. the eigenvalue of $\hat{J}_{Z}$.

The $\left\langle\hat{J}_{\gamma}{ }^{2}\right\rangle$-values which are of relevance here were calculated numerically from the known rotational constants and are listed in Table 1. The observed Zeeman shifts (see Table 2) correspond to differences of equations such as (4). Thus a set of linear equations for the $g$-values and susceptibility anisotropies is obtained with the observed Zeemanshifts as lefthand sides.

A standard least squares fit then leads to the $g$-values and susceptibility anisotropies listed in Table 3. For comparison the Zeeman shifts calculated according to (4) from the values given in Tables 3 and 1 are also given. The differences between the experimental and calculated Zeeman
Table 1. Numerical values for some low $J$ rigid rotor energy levels and the corresponding expectation values for the squared angular momentum operators divided by $\hbar^{2}$. For the calculation we used the rigid rotor Hamiltonian in Ray's form:

$$
\hat{\mathscr{H}}_{\text {rot }}=\frac{A+C}{2} \widehat{J}^{2}+\frac{A-C}{2} \hat{E}
$$

with $\hat{E}=\widehat{J}_{a}^{2}+\varkappa \widehat{J}_{b}{ }^{2}-\hat{J}_{c}^{2} ; \varkappa=(2 B-A-C) /(A-C)$ and the rotational constants $A=5.663720 \mathrm{GHz}, B=2.570624$ $\mathrm{GHz}$ and $C=1.767916 \mathrm{GHz}[20]$.

The matrix of $\hat{E}$ was set up within the symmetric top basis and was diagonalized numerically by a Jacobi procedure. The expectation values were calculated as [21]:

$$
\begin{aligned}
\left\langle\hat{J}_{b}^{2}\right\rangle= & \frac{\partial E_{J, K_{-} K_{+}(\varkappa)}}{\partial \varkappa}, \\
\left\langle\hat{J}_{c}^{2}\right\rangle= & {\left[J(J+1)-E_{J, K_{-} K_{+}(\varkappa)}\right.} \\
& \left.-(1-\varkappa) \frac{\partial E_{J, K_{-} K_{+}(\varkappa)}}{\partial \varkappa}\right] / 2, \\
\left\langle\hat{J}_{a}^{2}\right\rangle= & {\left[J(J+1)+E_{J, K_{-} K_{+}(\varkappa)}\right.} \\
& \left.-(1+\varkappa) \frac{\partial E_{J, K-K_{+}}(\varkappa)}{\partial \varkappa}\right] / 2,
\end{aligned}
$$

\begin{tabular}{|c|c|c|c|c|c|}
\hline$J$ & $K_{-} K_{+}$ & $\begin{array}{l}\text { rigid rotor } \\
\text { energy } \\
\text { in } \mathrm{GHz}\end{array}$ & $\left\langle\widehat{J}_{a}^{2}\right\rangle$ & $\left\langle\hat{J}_{b}{ }^{2}\right\rangle$ & $\left\langle\widehat{J}_{c}^{2}\right\rangle$ \\
\hline 0 & 0 & 0.000000 & 0.000000 & 0.000000 & 0.000000 \\
\hline 1 & 0 & 4.338540 & 0.000000 & 1.000000 & 1.000000 \\
\hline 1 & 1 & 7.431636 & 1.000000 & 0.000000 & \\
\hline 1 & 0 & 8.234344 & 1.000000 & 1.000000 & 0.000000 \\
\hline 2 & 0 & 12.878670 & 0.038438 & 2.642839 & 3.318723 \\
\hline 2 & 2 & 15.3060 & & & 4.000000 \\
\hline 2 & 1 & 17.714132 & 1.00 & 4.0 & 1.000000 \\
\hline & 1 & 26.993420 & & & \\
\hline 2 & 0 & 27.130370 & 3.961562 & 1.357161 & $0.68127 ?$ \\
\hline 3 & 3 & 25.370968 & 0.172639 & 4.339573 & 7.487788 \\
\hline & 1 & 96 & 1.02 & 2.3 & .6790 \\
\hline & 1 & & & & 2.730265 \\
\hline & 2 & 40.009040 & 4.000000 & 4.000 & 4.000000 \\
\hline 3 & 2 & 40.6 & 3.82 & 5.66 & 2.512212 \\
\hline & 3 & & & & 1.320961 \\
\hline 3 & 0 & 57.575522 & 8.970708 & 1.759557 & 1.269735 \\
\hline & 0 & 41.543452 & 0.421222 & 5.661005 & 13.917773 \\
\hline & 1 & & & 3.733 & \\
\hline & 1 & 50.476768 & 1.134528 & 13.328071 & 5.5374 \\
\hline & 3 & 57.255914 & 4.030546 & 7.718097 & 8.251357 \\
\hline & 2 & 59.096528 & 3.610471 & 12.049 & 4.339666 \\
\hline & $\begin{array}{ll}3 & 2\end{array}$ & 75.149801 & 8.9228 & 6.266 & 4.811116 \\
\hline & 3 & 75.252072 & 8.865472 & 6.671 & \\
\hline 4 & 1 & 99.403886 & 15.969454 & 2.281903 & 1.748643 \\
\hline & 0 & 99.405220 & 15.968307 & 2.289132 & 1.742561 \\
\hline
\end{tabular}

where $E J, K_{-} K_{+}(\varkappa)$ are the eigenvalues of the $\hat{E}$-matrix. The derivatives $\partial E J, K_{-} K_{+}(x) / \partial \varkappa$ were approximated as

$$
\left(E_{J, K_{-} K_{+}}(\varkappa+\Delta \varkappa)-E_{\left.J, K_{-} K_{+}(\varkappa)\right) / \Delta \varkappa}\right.
$$

with $\Delta \varkappa=10^{-6}$. Double precision calculation on a Digital PDP 10 computer (i.e. 16 significant figures) was used throughout. 
Table 2. Observed and calculated Zeeman shifts in fluorobenzene. The given field strengths are average values, averaged over the effective length of the absorption cell (= length of the Stark septum).

The complete calculated spectra are given in column 3 even though only the satellite frequencies listed in column 4 were used in the least squares procedure. In some cases also peaks assigned to unresolved satellites were included which are well reproduced by the intensity weighted means of the constituent satellites.

Frequency shifts are defined as $\Delta v=v(H)-v(0)$.

\begin{tabular}{|c|c|c|c|c|c|c|c|}
\hline $\begin{array}{l}\text { Rotational transition } \\
\text { zero field frequency } \\
\text { magnetic fieldstrength }\end{array}$ & & $M \rightarrow M^{\prime}$ & $\begin{array}{l}\text { Int. } \\
\text { (arbi- } \\
\text { trary } \\
\text { units) }\end{array}$ & $\begin{array}{l}\Delta \nu_{\text {calc }} \\
\mathrm{kHz}\end{array}$ & $\begin{array}{l}\Delta v_{\text {calc }} \\
\mathrm{kHz} \\
\text { intensity- } \\
\text { weighted }\end{array}$ & $\begin{array}{l}\Delta v_{\exp } \\
\mathrm{kHz}\end{array}$ & $\left(\Delta v_{\exp }-\Delta v_{\text {cale }}\right)$ \\
\hline $\begin{array}{c}2_{02} \rightarrow 3_{03} \\
12492.327 \mathrm{MHz} \\
18.898 \mathrm{kG}\end{array}$ & $\begin{array}{r}2 \\
1 \\
-2 \\
0 \\
-1\end{array}$ & $\begin{array}{r}2 \\
1 \\
-2 \\
0 \\
-1\end{array}$ & $\begin{array}{l}5 \\
8 \\
5 \\
9 \\
8\end{array}$ & $\left.\begin{array}{r}-432.4 \\
-200.7 \\
-167.8 \\
-\quad 79.3 \\
-\quad 68.4\end{array}\right\}$ & $\begin{array}{r}-432.4 \\
-188.0 \\
-\quad 74.2\end{array}$ & $\begin{array}{l}-433.5 \\
-188.1 \\
-\quad 73.7\end{array}$ & $\begin{array}{r}-1.1 \\
-0.1 \\
0.5\end{array}$ \\
\hline $\begin{array}{c}2_{02} \rightarrow 3_{03} \\
12492.327 \mathrm{MHz} \\
18.874 \mathrm{kG}\end{array}$ & $\begin{array}{r}2 \\
-2 \\
1 \\
-1 \\
0 \\
0 \\
2 \\
1 \\
-1 \\
-2\end{array}$ & $\begin{array}{r}1 \\
-1 \\
0 \\
0 \\
1 \\
-1 \\
3 \\
2 \\
-2 \\
-3\end{array}$ & $\begin{array}{r}2 \\
2 \\
6 \\
6 \\
12 \\
12 \\
30 \\
20 \\
20 \\
30\end{array}$ & $\left.\begin{array}{r}-710.2 \\
-456.1 \\
-289.8 \\
-167.8 \\
10.4 \\
20.6 \\
36.5 \\
78.5 \\
220.8 \\
311.0\end{array}\right\}$ & $\begin{array}{r}-710.2 \\
-456.1 \\
-289.8 \\
-167.8 \\
\\
27.2 \\
\\
78.5 \\
220.8 \\
311.0\end{array}$ & $\begin{array}{r}-710.2 \\
-449.1 \\
-291.3 \\
-170.2 \\
30.3 \\
\\
81.3 \\
223.2 \\
313.2\end{array}$ & $\begin{array}{r}0.0 \\
7.0 \\
-1.5 \\
-2.4 \\
\\
3.1 \\
\\
2.8 \\
2.4 \\
2.3\end{array}$ \\
\hline $\begin{array}{l}3_{13} \rightarrow 4_{14} \\
15513.774 \mathrm{MHz} \\
18.874 \mathrm{kG}\end{array}$ & $\begin{array}{r}3 \\
2 \\
-3 \\
1 \\
-2 \\
0 \\
-1\end{array}$ & $\begin{array}{r}3 \\
2 \\
-3 \\
1 \\
-2 \\
0 \\
-1\end{array}$ & $\begin{array}{r}7 \\
12 \\
7 \\
15 \\
12 \\
16 \\
15\end{array}$ & $\left.\begin{array}{r}-601.1 \\
-317.2 \\
-315.3 \\
-127.9 \\
-126.7 \\
-\quad 33.0 \\
-\quad 32.6\end{array}\right\}$ & $\begin{array}{l}-601.1 \\
-316.5 \\
-127.4 \\
-\quad 32.8\end{array}$ & $\begin{array}{l}-601.3 \\
-317.4 \\
-127.1 \\
-\quad 30.1\end{array}$ & $\begin{array}{r}-0.2 \\
-0.9 \\
0.3 \\
2.7\end{array}$ \\
\hline $\begin{array}{c}3_{13} \rightarrow 4_{14} \\
15513.774 \mathrm{MHz} \\
18.874 \mathrm{kG}\end{array}$ & $\begin{array}{r}-1 \\
3 \\
2 \\
1 \\
-1 \\
-3 \\
-2\end{array}$ & $\begin{array}{r}0 \\
4 \\
3 \\
2 \\
-2 \\
-4 \\
-3\end{array}$ & $\begin{array}{l}12 \\
56 \\
42 \\
30 \\
30 \\
56 \\
42\end{array}$ & $\left.\begin{array}{r}-237.7 \\
-140.2 \\
-\quad 22.9 \\
0.0 \\
339.0 \\
389.3 \\
411.4\end{array}\right\}$ & $\begin{array}{r}-237.7 \\
-140.2 \\
-\quad 13.4 \\
339.0 \\
398.7\end{array}$ & $\begin{array}{r}-226.9 \\
-136.5 \\
-\quad 13.3 \\
343.5 \\
399.9\end{array}$ & $\begin{array}{l}0.1 \\
4.5 \\
1.2\end{array}$ \\
\hline $\begin{array}{c}2_{11} \rightarrow 3_{12} \\
14125.550 \mathrm{MHz} \\
18.898 \mathrm{kG}\end{array}$ & $\begin{array}{r}1 \\
0 \\
2 \\
-1 \\
-2\end{array}$ & $\begin{array}{r}1 \\
0 \\
2 \\
-1 \\
-2\end{array}$ & $\begin{array}{l}8 \\
9 \\
5 \\
8 \\
5\end{array}$ & $\left.\begin{array}{r}-\quad 97.5 \\
-\quad 95.9 \\
80.2 \\
85.2 \\
445.6\end{array}\right\}$ & $\begin{array}{r}-\quad 96.6 \\
83.3 \\
445.6\end{array}$ & $\begin{array}{r}-\quad 96.0 \\
83.2 \\
446.6\end{array}$ & $\begin{array}{r}-0.1 \\
1.0\end{array}$ \\
\hline $\begin{array}{c}2_{11} \rightarrow 3_{12} \\
14125.550 \mathrm{MHz} \\
18.874 \mathrm{kG}\end{array}$ & $\begin{array}{r}0 \\
1 \\
-1 \\
-2 \\
1 \\
0 \\
2 \\
-1\end{array}$ & $\begin{array}{r}-1 \\
0 \\
-2 \\
-3 \\
2 \\
1 \\
3 \\
0\end{array}$ & $\begin{array}{r}12 \\
6 \\
20 \\
30 \\
20 \\
12 \\
30 \\
6\end{array}$ & $\left.\begin{array}{r}-547.4 \\
-465.9 \\
-450.0 \\
-173.8 \\
187.8 \\
272.9 \\
281.7 \\
536.9\end{array}\right\}$ & $\begin{array}{r}-547.4 \\
-453.7 \\
-173.8 \\
187.8 \\
279.1 \\
536.9\end{array}$ & $\begin{array}{r}-549.1 \\
-454.1 \\
-173.7 \\
190.6 \\
279.7 \\
534.3\end{array}$ & $\begin{array}{r}-1.7 \\
-0.4 \\
0.1 \\
2.8 \\
0.6 \\
-2.6\end{array}$ \\
\hline $\begin{array}{c}3_{03} \rightarrow 4_{04} \\
16172.488 \mathrm{MHz} \\
18.898 \mathrm{kG}\end{array}$ & $\begin{array}{r}3 \\
2 \\
1 \\
-3 \\
0 \\
-2 \\
-1\end{array}$ & $\begin{array}{r}3 \\
2 \\
1 \\
-3 \\
0 \\
-2 \\
-1\end{array}$ & $\begin{array}{r}7 \\
12 \\
15 \\
7 \\
16 \\
12 \\
15\end{array}$ & $\left.\begin{array}{r}-513.7 \\
-322.5 \\
-180.6 \\
-107.5 \\
-\quad 88.2 \\
-\quad 51.6 \\
-\quad 45.2\end{array}\right\}$ & $\begin{array}{r}-513.7 \\
-322.5 \\
-180.6 \\
-\quad 94.1 \\
-\quad 48.1\end{array}$ & $\begin{array}{r}-516.0 \\
-320.5 \\
-179.4 \\
-\quad 93.1 \\
-\quad 53.2\end{array}$ & $\begin{array}{r}-2.3 \\
2.0 \\
1.2 \\
1.0\end{array}$ \\
\hline
\end{tabular}


Table 2 (contin.)

\begin{tabular}{|c|c|c|c|c|c|c|c|}
\hline $\begin{array}{l}\text { Rotational transition } \\
\text { zero field frequency } \\
\text { magnetic fieldstrength }\end{array}$ & & $M \rightarrow M^{\prime}$ & $\begin{array}{l}\text { Int. } \\
\text { (arbi- } \\
\text { trary } \\
\text { units) }\end{array}$ & $\begin{array}{l}\Delta v_{\text {calc }} \\
\mathrm{kHz}\end{array}$ & $\begin{array}{l}\Delta v_{\text {calc }} \\
\mathrm{kHz} \\
\text { intensity- } \\
\text { weighted }\end{array}$ & $\begin{array}{l}\Delta v_{\exp } \\
\mathrm{kHz}\end{array}$ & $\left(\Delta v_{\exp } \underset{\mathrm{kHz}}{\left.-\Delta v_{\mathrm{cal}} \mathrm{c}\right)}\right.$ \\
\hline $\begin{array}{c}3_{03} \rightarrow 4_{04} \\
16172.488 \mathrm{MHz} \\
18.874 \mathrm{kG}\end{array}$ & $\begin{array}{r}-1 \\
1 \\
3 \\
0 \\
2 \\
1 \\
0 \\
-1 \\
-2 \\
-3\end{array}$ & $\begin{array}{r}0 \\
0 \\
4 \\
1 \\
3 \\
2 \\
-1 \\
-2 \\
-3 \\
-4\end{array}$ & $\begin{array}{l}12 \\
12 \\
56 \\
20 \\
42 \\
30 \\
20 \\
30 \\
42 \\
56\end{array}$ & $\left.\begin{array}{r}-187.7 \\
-177.5 \\
-\quad 95.6 \\
-\quad 90.7 \\
-\quad 44.7 \\
-\quad 43.1 \\
54.7 \\
237.6 \\
371.2 \\
455.5\end{array}\right\}$ & $\begin{array}{r}-182.6 \\
-\quad 94.3 \\
-\quad 44.0 \\
54.7 \\
237.6 \\
371.2 \\
455.5\end{array}$ & $\begin{array}{r}-181.5 \\
-\quad 93.3 \\
-\quad 43.6 \\
55.3 \\
241.4 \\
373.8 \\
454.6\end{array}$ & $\begin{array}{r}0.4 \\
0.6 \\
3.8 \\
2.6 \\
-0.9\end{array}$ \\
\hline $\begin{array}{c}3_{22} \rightarrow 4_{23} \\
17246.936 \mathrm{MHz} \\
18.898 \mathrm{kG}\end{array}$ & $\begin{array}{r}3 \\
2 \\
1 \\
0 \\
-1 \\
-2 \\
-3\end{array}$ & $\begin{array}{r}3 \\
2 \\
1 \\
0 \\
-1 \\
-2 \\
-3\end{array}$ & $\begin{array}{r}7 \\
12 \\
15 \\
16 \\
15 \\
12 \\
7\end{array}$ & $\begin{array}{r}-354.0 \\
-297.6 \\
-212.0 \\
-\quad 97.3 \\
46.7 \\
219.8 \\
422.1\end{array}$ & $\begin{array}{r}-354.0 \\
-297.6 \\
-212.0 \\
-\quad 97.3 \\
46.7 \\
219.8 \\
422.1\end{array}$ & $\begin{array}{r}-351.9 \\
-297.9 \\
-213.3 \\
-\quad 97.2 \\
46.8 \\
221.4 \\
426.6\end{array}$ & $\begin{array}{r}2.1 \\
-0.3 \\
-1.3 \\
0.1 \\
0.1 \\
1.6 \\
4.5\end{array}$ \\
\hline $\begin{array}{c}3_{22} \rightarrow 4_{23} \\
17246.936 \mathrm{MHz} \\
20.825 \mathrm{kG}\end{array}$ & $\begin{array}{r}2 \\
1 \\
0 \\
-1 \\
-2 \\
3 \\
2 \\
1 \\
0 \\
-3 \\
-1\end{array}$ & $\begin{array}{r}1 \\
0 \\
-1 \\
-2 \\
-3 \\
4 \\
3 \\
2 \\
1 \\
-4 \\
0\end{array}$ & $\begin{array}{r}6 \\
12 \\
20 \\
30 \\
42 \\
56 \\
42 \\
30 \\
20 \\
56 \\
12\end{array}$ & $\left.\begin{array}{r}-688.8 \\
-564.0 \\
-403.7 \\
-208.1 \\
23.1 \\
41.1 \\
59.6 \\
113.5 \\
202.9 \\
289.6 \\
327.7\end{array}\right\}$ & $\begin{array}{r}-688.8 \\
-564.0 \\
-403.7 \\
-208.1 \\
41.2 \\
113.5 \\
202.9\end{array}$ & $\begin{array}{l}-686.5 \\
-562.9 \\
-396.9 \\
-202.5\end{array}$ & $\begin{array}{l}2.3 \\
1.1 \\
6.8 \\
5.6\end{array}$ \\
\hline
\end{tabular}

shifts are below $\pm 10 \mathrm{kHz}$ which corresponds to our experimental accuracy and confirms the validity of the first order energy expression given in (4).

The experimental $g$-values and susceptibilities may now be inserted into the corresponding theoretical expressions [11] (5) and (6) to give the molecular electric quadrupole moments and the (7) anisotropies in the second moments of the electron charge distribution (8). These derived quantities are given in Table 4. Also given in Table 4 are the individual elements of the magnetic susceptibility tensor. They require the average magnetic susceptibility as additional input data which was taken from the literature [12].

$$
\begin{aligned}
& g_{a a}=\frac{m_{\mathrm{p}}}{I_{a a}}\left(\sum_{\nu}^{\text {nuclei }} Z_{\nu}\left(b_{\nu}^{2}+c_{\nu}^{2}\right)+\frac{2}{m}\right. \\
& \left.\cdot \sum_{n}^{\substack{\text { excited } \\
\text { states }}} \frac{\left|\left\langle n\left|\frac{\hbar}{i} \sum_{\varepsilon}^{\text {electrons }} b_{\varepsilon} \frac{\partial}{\partial c_{\varepsilon}}-c_{\varepsilon} \frac{\partial}{\partial b_{\varepsilon}}\right| 0\right\rangle\right|^{2}}{E_{0}-E_{n}}\right),
\end{aligned}
$$

Table 3. Nonvanishing $g$-tensor elements and the anisotropies in the diagonal elements of the molecular magnetic susceptibility tensor.

The susceptibilities are given in units of $10^{-6} \mathrm{erg} /\left(\mathrm{G}^{2} \mathrm{~mol}\right)$. They follow from a least squares fit according to (4) and the values of Tables 1 and 2. Only the relative signs of the $g$-values are determined experimentally, but the opposite choice of sign would lead to unreasonable large values for the quadrupole moments in Table 4 and to a negative sign of the electronic ground state expectation value

$$
\left\langle 0\left|\sum_{\varepsilon} c_{\varepsilon}^{2}\right| 0\right\rangle \text {. }
$$

It can thus be discarded.

The bulk susceptibility was taken from the literature [12]. For it a $\pm 5 \cdot 10^{-6} \mathrm{erg} /\left(\mathrm{G}^{2} \mathrm{~mol}\right)$ uncertainty is assumed to account for possible differences between gas phase and liquid phase susceptibilities.

\begin{tabular}{lcl}
\hline & Value & Standard deviation \\
\hline$g_{a a}$ & -0.068920 & \pm 0.00022 \\
$g_{b b}$ & -0.041606 & \pm 0.00013 \\
$g_{c c} \chi_{a a}-\chi_{b b}-\chi_{c c}$ & 51.68 & \pm 0.00009 \\
$2 \chi_{b b}-\chi_{c c}-\chi_{a a}$ & 61.69 & \pm 0.26 \\
$\left(\chi_{a a}+\chi_{b b}+\chi_{c c}\right) / 3$ & -58.4 & \pm 5 \\
\hline
\end{tabular}


$m_{\mathrm{p}}=$ proton mass, $m=$ electron mass,
$E_{n}=n$-th electronic eigenvalue in the infinite nuclear mass approximation.

$$
\begin{aligned}
& \chi_{a a}=-\frac{e^{2}}{4 m c^{2}}\left(\left\langle 0\left|\sum_{\varepsilon} b_{\varepsilon}^{2}+c_{\varepsilon}^{2}\right| 0\right\rangle+\frac{2}{m} \sum_{n}^{\text {electrons }} \frac{\substack{\text { excited } \\
\text { states }}}{\left|\left\langle n\left|\frac{\hbar}{i} \sum_{3}^{\text {electrons }} b_{\varepsilon} \frac{\partial}{\partial c_{\varepsilon}}-c_{\varepsilon} \frac{\partial}{\partial b_{\varepsilon}}\right| 0\right\rangle\right|^{2}} E_{0}-E_{n}\right), \\
& c=\text { velocity of light, } \\
& Q_{a a}=-\frac{\hbar e}{8 \pi m_{\mathrm{p}}}\left\{\frac{2 g_{a a}}{A}-\frac{g_{b b}}{B}-\frac{g_{c c}}{C}\right\}-\frac{2 m c^{2}}{e}\left\{2 \chi_{a a}-\chi_{b b}-\chi_{c c}\right\}, \\
& \left\langle 0\left|\sum_{\varepsilon}^{\text {electrons }}\left(b_{\varepsilon}{ }^{2}-c_{\varepsilon}^{2}\right)\right| 0\right\rangle=\sum_{v}^{\text {nuclei }} Z_{\nu}\left(b_{\nu}^{2}-c_{\nu}^{2}\right)+\frac{h}{8 \pi^{2} m_{\mathrm{p}}}\left(\frac{g_{b b}}{B}-\frac{g_{c c}}{C}\right) \\
& +\frac{4 m c^{2}}{3 e^{2}}\left\{\left(2 \chi_{b b}-\chi_{a a}-\chi_{c c}\right)-\left(2 \chi_{c c}-\chi_{a a}-\chi_{b b}\right)\right\} \text {. }
\end{aligned}
$$

\section{Discussion}

In our discussion we will concentrate on the molecular quadrupole moment $Q_{c c}$ and on the out of plane component of the so called nonlocal magnetic susceptibility.

Table 4. Molecular parameters derived from the data presented in Table 3, from the bulk susceptibility [12] and from the molecular structure [20]. As discussed in Ref. [6] the quadrupole moments determined here via (7) of the text should come close to the vibronic ground state expectation values. Also listed for comparison are molecular electric quadrupole moments and dipole moments calculated from CNDO/2 wavefunctions as described in Refs. $[15,16]$.

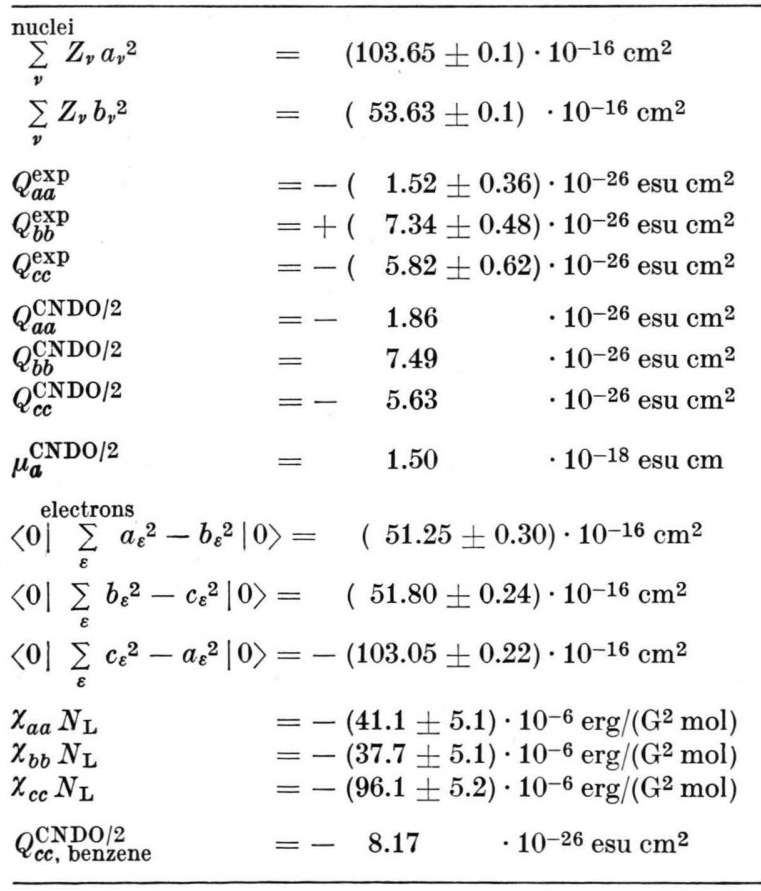

For the discussion of $Q_{c c}$ we first transform it to a coordinate system shifted along the $a$-axis by $0.49 \AA$ into the center of the aromatic ring:

$$
\begin{aligned}
& Q_{c c, \text { center }}=Q_{c c}^{\exp }+\mu_{a} \cdot \Delta a=-(5.82 \pm 0.62) \\
& \cdot 10^{-26} \mathrm{esu} \mathrm{cm}{ }^{2}+1.66 \cdot 10^{-18} \cdot 0.49 \cdot 10^{-8} \mathrm{esu} \mathrm{cm}^{2} \\
& =-(5.01 \pm 0.62) 10^{-26} \mathrm{esu} \mathrm{cm}^{2} .
\end{aligned}
$$

Here $\mu_{a}=(1.66 \pm 0.03) D$ is the molecular electric dipole moment determined from the Stark effect [13] and we have assumed that the fluorine atom determines the negative end of the molecule.

As discussed in a previous paper [14], the

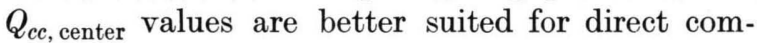
parison within the family of fluorosubstituted benzenes. We note that the revised value of $Q_{c c \text {, center }}$ almost exactly falls on the least squares straight line, which correlates the $Q_{c c \text {, center }}$ values to the number of fluorine substituents (see Fig. 7 of Ref. [14]). In our opinion this result lends further credit to the linear correlation between $Q_{c c \text {, center }}$ and the number of fluorine substituents.

Next we use (2) to deduce the molecular quadrupole moment of benzene from the $Q_{b b}$ and $Q_{c c}$-values of Table 3 . The new value is: $Q_{c c}$, benzene $=-8.8 \cdot 10^{-26} \mathrm{esu} \mathrm{cm}^{2}$. The experimental uncertainties in the fluorobenzene values lead to an experimental uncertainty of $\pm 0.9 \cdot 10^{-26} \mathrm{esu}^{2} \mathrm{~cm}^{2}$ in this value. In order to estimate the additional uncertainty introduced by partial breakdown of assumption b) of Shoemaker and Flygare, we have calculated the molecular electric quadrupole moments for fluorobenzene and benzene from $\mathrm{CNDO} / 2$ wavefunctions as described in Refs. $[15,16]$. They too are listed in Table 4. 
If we combine the CNDO/2 $Q_{b b^{-}}$and $Q_{c c \text {-values }}$ for fluorobenzene according to (2) we get a predicted quadrupole moment for benzene: $Q_{c c}^{\text {pred }}=-8.74$ $\cdot 10^{-26} \mathrm{esu} \mathrm{cm}^{2}$. This value should be compared to the directly calculated value:

$$
Q_{c c}^{\mathrm{CNDO} / 2}=-8.17 \cdot 10^{-26} \mathrm{esu} \mathrm{cm}^{2} .
$$

Since CNDO/2 wavefunctions apparently work fairly well for the comparison of the molecular quadrupole moments of the closely related family of fluorosubstituted benzenes [14] we are confident that the difference of $0.5 \cdot 10^{-26} \mathrm{esu} \mathrm{cm}^{2}$, which corresponds to a $0.1 \AA^{2}$ difference in the $\langle 0| \sum_{\varepsilon} b_{\varepsilon}^{2}$ $-c_{\varepsilon}{ }^{2}|0\rangle$-values of benzene and fluorobenzene, is a reasonable estimate of the extrapolation uncertainty of (2). We even believe that the $\mathrm{CNDO} / 2$-calculations reproduce the sign of the extrapolation correction and that the true gas-phase value of $Q_{c c \text {, benzene }}$ should thus be slightly less negative than the extrapolated value. As final $Q_{c c^{-}}$value we therefore present:

$$
\begin{aligned}
Q_{c c, \text { benzene }} & =-(8.5 \pm 1.4) 10^{-26}{\mathrm{esu} \mathrm{cm}^{2}}^{2} \\
& =-(28.4 \pm 4.7) \cdot 10^{-40} \mathrm{C} \mathrm{m}^{2}
\end{aligned}
$$

where an extrapolation correction of +0.25 $\cdot 10^{-26} \mathrm{esu} \mathrm{cm}^{2}$ has been applied to the result of (2) and where the uncertainty limit of \pm 1.4 units is composed from the experimental uncertainty in the fluorobenzene quadrupole moments, \pm 0.9 units, and an extrapolation uncertainty of \pm 0.5 units.

We now turn to the out of plane component of the magnetic susceptibility tensor, $\chi_{c c}$. As has been discussed in detail by Schmalz et al. [17], $\chi_{c c}$ may be broken down into a "nonlocal" ( $\pi$-electron) ring current contribution and into a sum of local (atomic) contributions. With the local values from Table 5, Ref. [14], i.e. $\chi_{c c(\mathrm{H}-)}=-2.11, \chi_{c c\left(\mathrm{Csp}^{2}\right)}=$ -7.11 , and $\chi_{c c}(\mathrm{~F}-)=-5.6$, all in units of $10^{-6} \mathrm{erg} /$ $\left(\right.$ Gauss $\left.^{2} \mathrm{~mol}\right)$, we obtain $\chi_{c c}^{\text {local }}=-58.8 \cdot 10^{-6} \mathrm{erg} /$ (Gauss ${ }^{2}$ mol).

If we combine this value with the improved $\chi_{c c}$ value from Table $3, \chi_{c c}^{\text {nonlocal }}=-37.3 \cdot 10^{-6} \mathrm{erg} /$ (Gauss ${ }^{2} \mathrm{~mol}$ ) is obtained. We may now insert this value into Fig. 4 of Ref. [14], which shows a plot of $\chi_{c c}^{\text {nonlocal }}$ versus the calculated "CNDO/2- $\pi$ density alternation" for a whole family of fluorobenzenes and fluoropyridines. While the original value was about two units off, the revised value closely coincides with the least squares straight line which appears to correlate the $\chi_{c c}^{\text {nonlocal }}$ benzene-values and the corresponding " $\mathrm{CNDO} / 2-\pi$-density alternations". Thus this correlation certainly deserves further attention both experimentally and theoretically.

\section{Appendix}

Description of the high resolution Ku-Band superheterodyne bridge spectrometer.

As discussed earlier by Schwoch and Rudolph [18], the effects of $1 / f$ detectordiode noise and of signal oscillator noise can be reduced considerably by the use of a superheterodyne bridge spectrometer.

A detailed block diagram of our system is shown in Figure 2. At present all waveguide parts except for the sample cell and the reference cell are $\mathrm{Ku}$ band (12.4 to $18 \mathrm{GHz}$ ). The sample cell and reference cell are made from standard $J$-band brass waveguide (inner cross section $34.85 \cdot 15.80 \mathrm{~mm}^{2}$ ), to increase the effective absorption volume at given length and to reduce the effects of wall collision broadening.

Both cells contain a teflon supported Stark septum of $1.80 \mathrm{~m}$ length and may be cooled down simultaneously to approximately $-60^{\circ} \mathrm{C}$ by methanol flowing through cooling jackets.

Care has been taken to manufacture the cells as identical as possible.

The spectrometer needs two microwave sources, one as signal oscillator and one as local oscillator. In our setup the local oscillator is phaselocked to the signal oscillator at a $30 \mathrm{MHz}$ higher frequency.

In operation the frequency of the signal oscillator is first set to the center of the frequency range to be investigated and the phase shifters and waveguide attenuators in the sample- and reference arms are adjusted to minimize the microwave power which enters the detector arm (carrier suppression). (Residual carrier power is detected as a $30 \mathrm{MHz}$ IF-signal with the oscilloscope). Ideally complete destructive interference should be achieved between the microwave field directly propagating from the sample cell into the detector arm and the field coupled in from the reference arm via the $10 \mathrm{db}$ directional coupler. After this initial setting, the signal frequency and, phase locked to it, the local oscillator frequency is slowly swept through the 


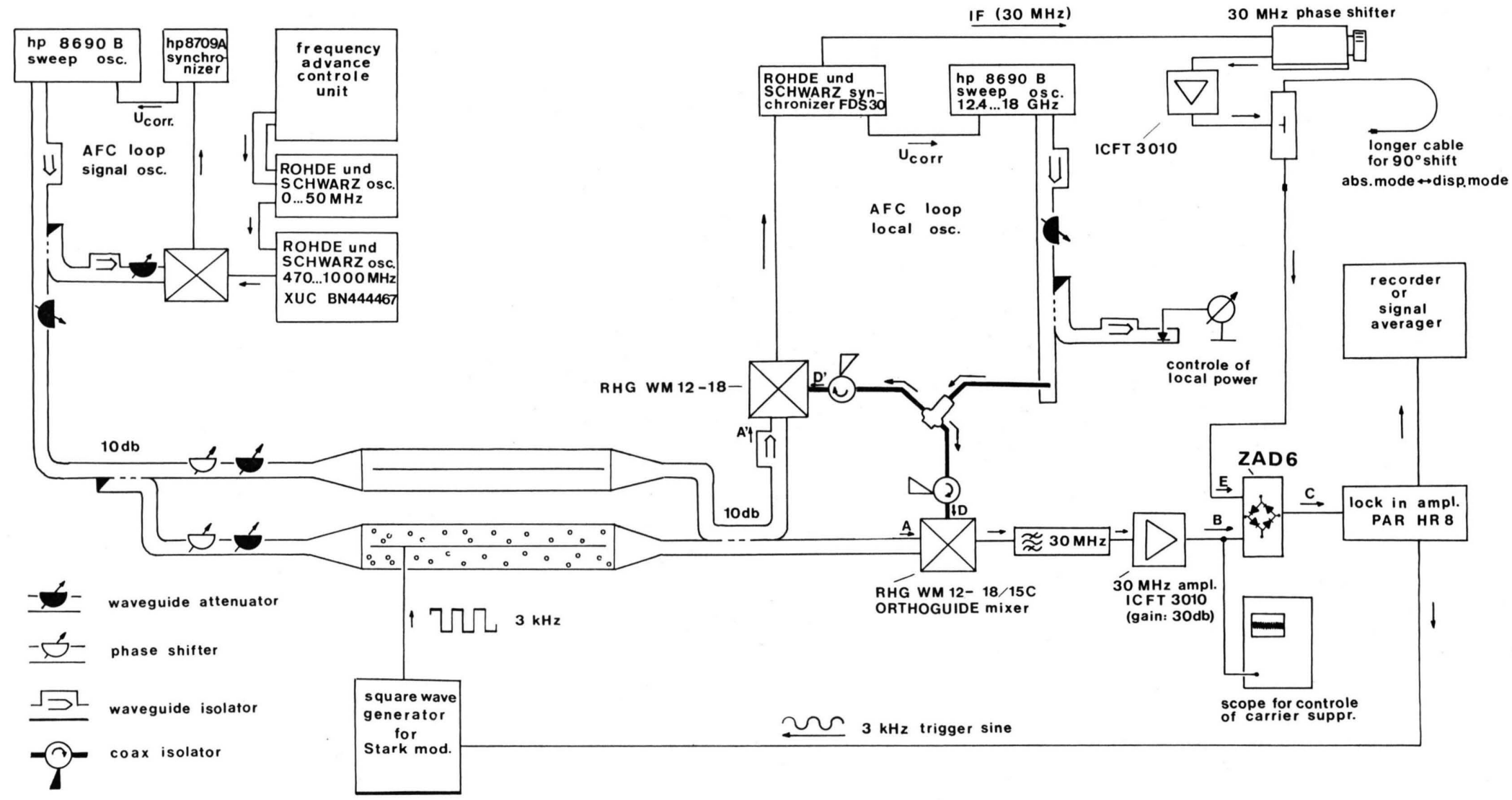

Fig. 2. Detailed block diagram of the Ku-band superheterodyne bridge spectrograph. In operation the phase shifters and attenuators are adjusted for complete destructive interference of the two microwaves recombined by the second $10 \mathrm{db}$ multihole directional coupler. In the presence of a molecular absorption however, a small signal of molecular origin propagates down to the orthoguide mixer, where it is heterodyned against a local oscillator radiation field $30 \mathrm{MHz}$ above (or below) the signal. After amplification the resulting IF-signal is heterodyned down to CW in the ZAD6 mixer to trace the molecular absorption/ dispersion curve depending on the phase setting of the $30 \mathrm{MHz}$ phase shifter. Standard $3 \mathrm{kHz}$ Stark-effect modulation is used in addition to minimize the effect of mechanical vibrations. (These cause a detuning (nonperfect carrier suppression) with low frequency noise characteristics.) 
frequency range. At frequencies in resonance (or close) to a molecular transition frequency, the bridge is slightly thrown out of balance and a small microwave signal of molecular origin reaches the double balanced mixer (RHG WM 12-18/15 C), where it is heterodyned down to an intermediate frequency of $30 \mathrm{MHz}$. After $50 \mathrm{db}$ amplification ( $20 \mathrm{db}$ in the integrated preamplifier of the mixer), the signal enters the RF port of a low frequency mixer (ZAD6 of MINI CIRCUITS Corporation).

With the corresponding reference frequency directly derived from the stabilization IF, residual phase noise of either the signal or (and) the local oscillator cancels in the final "dc-output" (see below). To reduce the effects of body noise etc. we use $3 \mathrm{kHz}$ Stark-effect modulation i.e. the molecular signal is chopped at a rate of $3 \mathrm{kHz}$ and so is that part of the ZAD6-output which is caused by the molecules. Standard phase sensitive detection at $3 \mathrm{kHz}$ is then used for a final improvement of the signal to noise ratio (PAR-HR 8).

In our experience the remaining noise in our instrument is dominated by local oscillator noise, which is not completely balanced out in the RHG mixer. We conclude this from the observation that temporary replacement of the backward wave tube oscillator by a POLARAD $1108 \mathrm{E}$ signal oscillator with a phase stabilized Klystron as microwave source did lead to an improvement of the signal to noise ratio by a factor of almost three (all power levels etc. were kept identical in this comparison). We intend to extend the operating range of the system to $\mathrm{X}$-band with a POLARAD source as local oscillator and we expect a further improved performance of the X-band system.

We may now mention the two effects which might cause problems in certain applications of the bridge system. One is, that sufficient carrier suppression may be obtained only over a limited bandwidth, typically on the order of $\pm 5 \mathrm{MHz}$ to $\pm 10 \mathrm{MHz}$ about the centerfrequency. The second effect is, that the presence of a dispersion signal may distort the overall profiles of partly resolved multiplets, which in turn might lead to misinterpretations.

We first discuss the bandwidth problem. Apparently it is associated with the use of oversized waveguide absorption cells, since it does not occur, if the cells are replaced by straight $\mathrm{Ku}$-band waveguide sections of equal length.
In oversized cells higher modes with different waveguide wavelengths, which might be generated for instance at the front end of the Starkplates or by an imperfect alignment of the tapered transitions, may propagate. Since the generation of such higher modes will in general be different in the two arms of the bridge and since the higher modes show stronger dispersion, they will cause a frequency dependent optical path difference and the bridge drifts out of balance with increasing frequency offset.

We now turn to the discussion of the "dispersionproblem". For better understanding we first give a simplified description of how the final output signal is generated.

To this end we assume that the IF-outputs of each of the three mixers are proportional to the low frequency part in the product of the corresponding incident RF- and local-signals. Then, if the signal oscillator frequency approaches the resonance frequency of a rotational transition it will start to polarize the molecular ensemble [19], and this polarization in turn leads to a molecular signal at the detector. This signal is essentially the same as in the classical model of an ensemble of damped harmonic oscillators which are dispersed in the absorption cell and which are driven by the incident microwave field. At the detector, the corresponding molecule-generated electric field strength may be written as:

$$
\begin{aligned}
\varepsilon(t)= & \varepsilon_{\mathrm{c}}(\omega) \cos (\omega t+\varphi) \\
& +\varepsilon_{\mathrm{s}}(\omega) \sin (\omega t+\varphi),
\end{aligned}
$$

where

$$
\varepsilon_{\mathrm{c}}(\omega) \approx-\frac{(2 \pi / \tau)}{\left(\omega_{0}-\omega\right)^{2}+(2 \pi / \tau)^{2}}
$$

corresponds to the "absorption signal", and where

$$
\varepsilon_{\mathrm{S}}(\omega) \approx-\frac{\left(\omega_{0}-\omega\right)}{\left(\omega_{0}-\omega\right)^{2}+(2 \pi / \tau)^{2}}
$$

corresponds to the "dispersion signal".

In these relations $\omega=2 \pi \nu$ corresponds to the signal oscillator frequency, $\omega_{0}=2 \pi \nu_{0}$ corresponds to the resonance frequency of the molecular transition, and $\tau$ corresponds to the molecular relaxation time ( $\tau=T_{1}=T_{2}$ assumed).

Doppler broadening, mode structure and wall collisions are neglected in this simplified treatment. We now assume complete carrier suppression. Then 


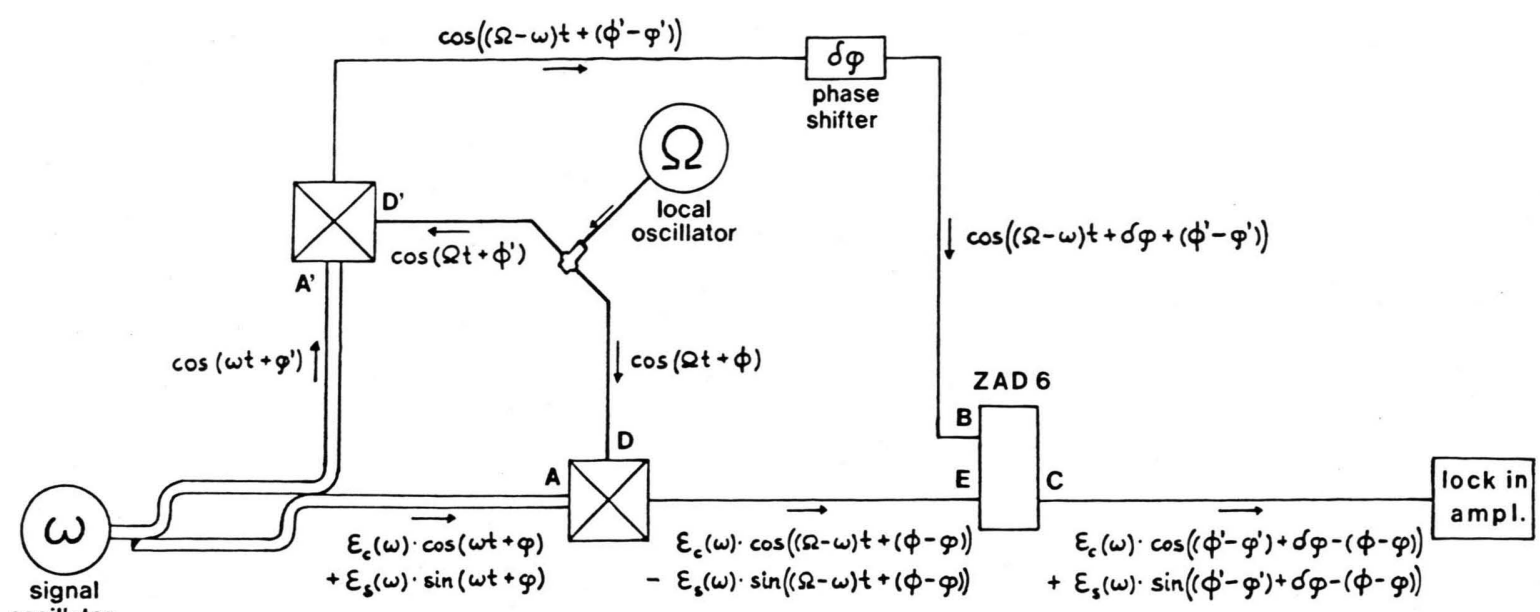

oscillator

Fig. 3. Signal flow in the Ku-band superheterodyne bridge spectrograph is illustrated in this simplified diagram. The local oscillator frequency is phase locked $30 \mathrm{MHz}$ above the signal oscillator frequency. Complete carrier suppression is assumed and it is assumed that the output of the mixers is proportional to the low frequency component in the product of the input signals. (At sufficiently high local levels it becomes independent on local power.) Depending on the $\delta \varphi$-phase setting, the ZAD6-output signal traces a linear combination of the absorption curve, $\varepsilon_{\mathrm{c}}(\omega)$, and the dispersion curve, $\varepsilon_{\mathrm{S}}(\omega)$. Ideally $\delta \varphi$ is set to minimize the $\varepsilon_{\mathrm{S}}(\omega)$ contribution. Improper phase setting causes problems in the analysis of partly resolved multiplets. However in well resolved multiplets an improper phase setting simply leads to a shift of all lines by the same amount without any effects on the splittings. In our experience the frequency dependence in the argument of the cosine and sine is sufficiently low so that the dispersion/absorption ratio does not change measurably over $a \pm 10 \mathrm{MHz}$ band.

only the molecular signal given in (A.1) is heterodyned in the MW $12-18 / 15 \mathrm{C}$ mixer against the local oscillator signal which is proportional to $\cos (\Omega t+\Phi)$ with $\Omega=\omega+2 \pi \cdot 3 \cdot 10^{7} \mathrm{~Hz}$. As a result an IF-signal proportional to

$$
\begin{aligned}
\varepsilon_{\mathrm{c}}(\omega) & \cdot \cos ((\Omega-\omega) t+(\Phi-\varphi)) \\
& -\varepsilon_{\mathrm{S}}(\omega) \cdot \sin ((\Omega-\omega) t+(\Phi-\varphi))
\end{aligned}
$$

will hit the ZAD 6-mixer at port $\mathrm{B}$ of the block diagram.

As shown on the diagram in Fig. 3 this IF-signal is heterodyned against a reference signal, which is directly derived from the IF-output of the stabilization mixer. Since the phase of this reference signal may be deliberately set to any value with the $30 \mathrm{MHz}$ phase shifter, we may write it as

$$
\cos \left((\Omega-\omega) t+\left(\Phi^{\prime}-\varphi^{\prime}\right)+\delta \varphi\right) .
$$

The advantage of deriving this $30 \mathrm{MHz}$ reference signal directly from the stabilization IF becomes obvious now. Phase fluctuations in the local- (or signal-) oscillator output affect $\Phi$ and $\Phi^{\prime}$ (or $\varphi$ and $\left.\varphi^{\prime}\right)$ simultaneously and cancel in the final output signal. The latter is proportional to

$$
\begin{aligned}
& \varepsilon_{\mathrm{c}}(\omega) \cos \left(\left(\Phi^{\prime}-\varphi^{\prime}\right)+\delta \varphi-(\Phi-\varphi)\right) \\
& \quad+\varepsilon_{\mathrm{s}}(\omega) \sin \left(\left(\Phi^{\prime}-\varphi^{\prime}\right)+\delta \varphi-(\Phi-\varphi)\right) .
\end{aligned}
$$

Depending on the $\delta \varphi$-setting, either the sine- or the cosine-term may be set to zero as is demonstrated in Figure 4. In standard operation the phase $\delta \varphi$ is

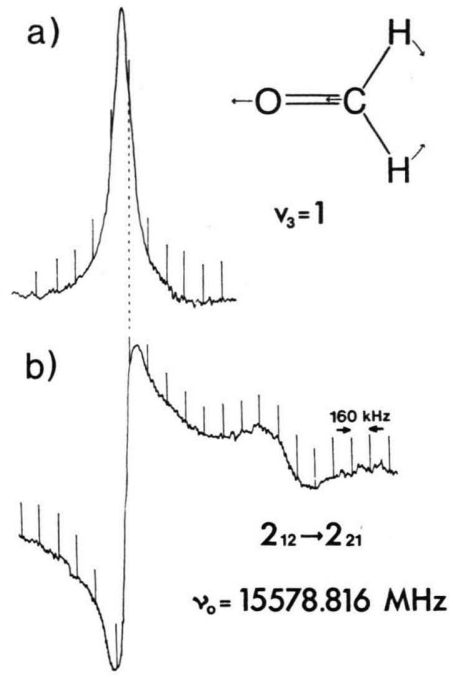

Fig. 4. Depending on the phase setting of the $30 \mathrm{MHz}$ phase shifter, the ZAD-6 output (see also Figs. 2 and 3) traces the absorption curve, $\varepsilon_{\mathbf{c}}(\omega)$, or the dispersion curve $\varepsilon_{\mathbf{S}}(\omega)$, as is demonstrated here with the $2_{12} \rightarrow 2_{21}$ transition of formaldehyde in its first excited state of the $\nu_{3}$-vibration $(\mathrm{H}-\mathrm{C}-\mathrm{H}$ bending mode). In case a) the phase in the trigonometric functions in the output signal was adjusted to zero. In case b) it was adjusted to $-90^{\circ}$. In the dispersion curve also a Stark satellite is shown at right. 
set thus, that the output signal traces the absorption curve, $\varepsilon_{\mathrm{c}}(\omega)$. To set the phase properly a single line is recorded several times and the $\delta \varphi$ phase is adjusted until the line profile is symmetric. Here the second problem becomes obvious. While in the case of relatively strong lines the $\delta \varphi$-setting is done within minutes, it may be extremely time consuming in the case of weak lines which - even though within the sensitivity range of the spectrometer - need signal averaging for, say, 30 minutes. We note here, that the proper phase setting is essential, if only partly resolved multiplets are to be analyzed, since a superposition of asymmetric lines i.e. satellites with profiles containing dispersive contributions, may considerably distort the overall appearance of the corresponding multiplet pattern.

We note however, that in view of the higher sensitivity of the bridge spectrometer, as compared

[1] R. L. Shoemaker and W. H. Flygare, J. Chem. Phys. 51, 2988 (1969).

[2] W. Hüttner and W. H. Flygare, J. Chem. Phys. 50, 2863 (1969)

[3] J. Vrbancich and G. L. D. Ritchie, J.C.S. Faraday II, 76, 648 (1980).

[4] D. H. Sutter, Z. Naturforsch. 26a, 1644 (1971).

[5] W. H. Flygare and R. C. Benson, Mol. Phys. 20, 225 (1971).

[6] D. H. Sutter and W. H. Flygare, Top. in Current Chem. 63, 69 (1976).

[7] W. H. Flygare, Chem. Revs. 74, 653 (1974).

[8] W. Gordy and R. L. Cook, Microwave Molecular Spectra, Intersc. Publ. New York 1970.

[9] L. Engelbrecht and D. H. Sutter, Z. Naturforsch. 30a, 1265 (1975).

[10] W. Hüttner and W. H. Flygare, J. Chem. Phys. 47, 4137 (1967).

[11] Compare for instance. Chapt. IV of Ref. [6].

[12] Handbook of Chemistry and Physics, 62nd Ed. (1981), CRC Press, Inc. Boca Raton, Florida. to a standard Stark-spectrometer, the phase setting becomes time consuming only in the case of "really weak" absorption lines.

\section{Acknowledgement}

During the development stage of the spectrometer we had many helpful discussions with $\mathrm{H}$. Bomsdorf who has set up a bridge spectrometer for experiments in the time domain [22]. Further we express our gratitude to the workshop of our institute for the excellent craftmanship and we thank Professors H. Dreizler and J. Sheridan for critically reading the manuscript. All computer calculations were carried out at the computer center of the University of Kiel. This work was supported by the Deutsche Forschungsgemeinschaft under grants Su 41/9 and Su 41/11. Support from the Fonds der Chemische Industrie is also gratefully acknowledged.

[13] D. G. de Kowalewski, P. Kökeritz, and D. H. Selén, J. Chem. Phys. 31, 1438 L (1959).

[14] D. Hübner, M. Stolze, and D. H. Sutter, Z. Naturforsch. 36a, 332 (1981).

[15] E. Hamer, PHD thesis, Kiel 1973.

[16] E. Hamer, L. Engelbrecht, and D. H. Sutter, Z. Naturforsch. 29 a, 924 (1974).

[17] T. G. Schmalz, C. L. Norris, and W. H. Flygare, J. Amer. Chem. Soc. 95, 7961 (1973).

[18] H. Rudolph and D. Schwoch, Z. angew. Phys. 31, 197 (1971).

[19] J. McGurck and W. H. Flygare, Adv. Chem. Phys. 25, 1 (1974).

[20] L. Nygaard, I. Bojesen, T. Pedersen, and J. RastrupAndersen, J. Mol. Structure 2, 200 (1968).

[21] L. D. Landau and E. M. Lifschitz, Lehrbuch der Theoretischen Physik, Vol. III, Quantenmechanik, Akademieverlag Berlin 1965, p. 38.

[22] H. Bomsdorf and H. Dreizler, Z. Naturforsch. 36a, 473 (1981). 